-

\title{
An Overview of Image Analysis Algorithms for License Plate Recognition
}

\author{
Khalid ABOURA ${ }^{1}$, Rami AL-HMOUZ² \\ ${ }^{1}$ University of Dammam, College of Business Administration, Dammam, Saudi Arabia \\ kaboura@ud.edu.sa \\ ${ }^{2}$ King Abdulaziz University, Department of Electrical and Computer Engineering, Jeddah, Saudi Arabia \\ ralhmouz@kau.edu.sa
}

\begin{abstract}
Background and purpose: We explore the problem of License Plate Recognition (LPR) to highlight a number of algorithms that can be used in image analysis problems. In management support systems using image object recognition, the intelligence resides in the statistical algorithms that can be used in various LPR steps. We describe a number of solutions, from the initial thresholding step to localization and recognition of image elements. The objective of this paper is to present a number of probabilistic approaches in LPR steps, then combine these approaches together in one system. Most LPR approaches used deterministic models that are sensitive to many uncontrolled issues like illumination, distance of vehicles from camera, processing noise etc. The essence of our approaches resides in the statistical algorithms that can accurately localize and recognize license plate.

Design/Methodology/Approach: We introduce simple and inexpensive methods to solve relatively important problems, using probabilistic approaches. In these approaches, we describe a number of statistical solutions, from the initial thresholding step to localization and recognition of image elements. In the localization step, we use frequency plate signals from the images which we analyze through the Discrete Fourier Transform. Also, a probabilistic model is adopted in the recognition of plate characters. Finally, we show how to combine results from bilingual license plates like Saudi Arabia plates.

Results: The algorithms provide the effectiveness for an ever-prevalent form of vehicles, building and properties management. The result shows the advantage of using the probabilistic approached in all LPR steps. The averaged classification rates when using local dataset reached $79.13 \%$.

Conclusion: An improvement of recognition rate can be achieved when there are two source of information especially of license plates that have two independent texts.
\end{abstract}

\section{Keywords: Image Analysis; Probabilistic Modeling; Signal Processing; License Plate Recognition}

\section{Introduction}

Image processing transforms information from the real world into image data represented by matrices. The manipulation of these real valued matrices requires mathematical and statistical solutions in order to locate objects in the images. Optical character recognition (OCR) is the technology of identifying written or printed text that are included in images using image-processing algorithms. OCR is a well-explored problem. It has been used and commercialized in many applications that include LPR, libraries, banks, converting document into text searchable document in scanner and sorting mail in post offices. Some of our solutions apply in such contexts.

Many around the world contributed to research in license plate recognition. LPR systems are in use in several countries, and Britain has an extensive ANPR (Automatic

Received: January 21, 2017; revised: March 30, 2017; accepted: May 28, 2017 
Number Plate Recognition) CCTV network. Such achievements took many years of research. However, the problem remains difficult to solve perfectly. For example, in many countries the law still requires the plate number of an infringing car to be recognized visually. This reliability issue keeps the LPR problem an active research topic. LPR uses video captured images to automatically identify vehicles through their license plate. LPR finds applications in theft prevention, parking lot management, hotels and property management, traffic laws enforcement, border control etc. There are other methods to identify cars such as transponders, bar-coded labels and radio-frequency tags. License plate reading remains however the way a vehicle is identified. LPR attempts to make the reading automatic by processing sets of images captured by cameras. There are three steps in the process; detecting a vehicle, triggering the captures of images related to that vehicle and treating those images for recognition of the characters in the license plate. The capture of the images, their transfer in digital form to a processor and the coordination of all tasks in a LPR system is a feasible engineering problem. The processing of images for recognition is where research starts. LPR has three main parts; localization of license plate from image, segmentation of characters from localized license plate region and recognition of those characters. These steps are performed automatically by software and require algorithms.

\subsection{License Plate Localization}

Plate localization is the first step in LPR. It aims to locate the license plate of the vehicle in an image. A variety of approaches have been proposed to localize a license plate in captured video images (Al-Hmouz and Aboura, 2014). Some of the existing methods are morphological operations, edge detection, corner detection, sliding concentric windows (Anagnostopoulos et al., 2006), fuzzy logic (Chang et al., 2006 ), Hough transform (Duc et al.,2005), neural networks (Kim et al. 2000), Fourier transform (Acosta, 2004), adaptive boosting (AdaBoost) algorithm (Dlagnekov, 2004). Al-Hmouz and Aboura (2014) introduce a new approach of plate localization using a statistical analysis of Discrete Fourier Transform of the plate signal. The plate signal is represented by five statistics: strength of the signal, normalized maximum amplitude, frequency of maximum amplitude, frequency center and frequency spread. Combining with the color-based histogram thresholding (Aboura, 2008), the method achieves $97.27 \%$ accuracy using plate signals from binary images.

\subsection{License Plate Segmentation}

The second task in LPR is to extract the characters from the localized license plate region. The most common meth- od is the projection method. First, the image is thresholded, reduced from a color or gray-scale image to a black and white (background/foreground) image. The projection method then counts the number of foreground pixels vertically and horizontally in the license plate area to separate and extract the characters. Other methods and variants include, thin window scanning, local vector quantization, scale shape analysis, Laplacian transform, Hough transform and Markov Random fields. The final task in LPR is the recognition of characters. An optical character recognition algorithm is used to recognize characters in the image. A series of approaches were developed. The most common ones are the correlation-based template matching and neural networks. Other methods are feature based, use pattern mapping or are based on the Hausdorff distance. Binary classifiers are used as well as the Hidden Markov model and probabilistic modeling. All three steps in LPR rely on thresholding or binarization of the original image. A common approach is the well cited Otsu method (Otsu, 1979). The Otsu method performs rather poorly in LPR if applied to the whole image. The resulting binary image often doesn't show the license plate characters as foreground. In Aboura (2008), this topic is discussed and a new thresholding method is introduced. It is used in the approaches described in this article.

\subsection{License Plate Recognition}

The final task in LPR is the recognition of characters. The extraction of characters results in a number of selected regions in the image. These regions contain the characters of the license plate and are processed for recognition by what is referred to as an Optical Character Recognition (OCR) algorithm. While OCR applies in LPR, it is a field that has a much wider stage of applications. OCR dates back to 1929 and is a method designed to translate automatically images of handwritten or typewritten text. There are ofthe-shelves software packages that can translate a faxed page of text or an image that contains text. However, these OCR commercial packages do not yield good results in the case of LPR. A series of approaches were developed to recognize license plate characters. The most common OCR approaches used in LPR are the correlation-based template matching and neural networks. Other methods are feature based, use pattern mapping or are based on the Hausdorff distance. Binary classifiers are also used as well as the Hidden Markov model (Aboura and Al-Hmouz, 2007). Neural networks (NN) haven been applied successfully in many prediction, classification and recognition problems. In LPR, they are used to localize the license plate in the image and to recognize the extracted characters of the plate. A Neural network is an artificial network made up of sets of interconnected nodes called neurons. In its simple form, the feed forward structure used in LPR, there is a set of input nodes, for example the features and attributes of 
the image being processed (Figure 1), that are connected through a network of nodes, hidden layers, to a set of output nodes, the classes to which the image belongs.

Template Matching is the other approach most used in character recognition in LPR. It is a technique in image analysis for scanning an image template until part of it matches an image at hand. There are many variants in the application of template matching to character recognition. In its simplest form, the image in its binary form (Figure 1) is compared with same size parts of the template image using a suitable metric. The metric can be the Euclidian distance or a correlation measure between the pixels of the image and the template. For example, the cross correlation, a statistical measure used by Horowitz (1957) and Pratt (1974) for image recognition, can be a metric for template matching. The template matching approach is combined with other methods in character recognition. However, it remains a method based on the minimization of a distance between two images and can prove to be inefficient in practice.

The objective of this paper is to present a number of probabilistic approaches in LPR steps, then combine these approaches together in one system. Most LPR approaches used deterministic models that are sensitive to many uncontrolled issues like illumination, distance of vehicles from camera, processing noise etc. The essence of our approaches reside in the statistical algorithms that can accurately localize and recognize license plate.

The structure of the paper is as follows, first we show the probabilistic approaches that investigate the localization and recognition phases in LPR systems. In section 3, we show the results of the presented approaches. Followed by discussion in section 4 . Finally, the paper ends with conclusions in section 5 .

\section{Research Methods in Probabilistic Image Analysis}

In this section, we review some of the statistical and signal processing methods that address the localization and character recognition problems discussed in the introduction. We also point to the global thresholding method used in the methodology.

\subsection{Localization using the Discrete Fourier Transform signal}

A variety of approaches have been proposed to localize a license plate in captured video images. Al-Hmouz and Aboura (2014) introduce a new approach of plate localization using a statistical analysis of Discrete Fourier Transform of the plate signal. The plate signal is represented by five statistics: strength of the signal, normalized maximum amplitude, frequency of maximum amplitude, frequency center and frequency spread. The idea is that license plate numbers show frequencies that lead themselves to a spectral analysis through the Fourier transform. Al-Hmouz and Aboura (2014) introduce a formal statistical analysis of the Fourier transform data from a systematic scanning of the image. The authors use the Hysteresis thresholding (Canny, 1986) over scanned regions of the image to obtain a clear signal. They improve the speed of the methodology and its accuracy by thresholding the whole image using the binarization approach of Aboura (2008). The behavior of the power spectrum of the scanned region shows a significant increase in magnitude at some frequencies for scanned regions that contain the license plate or parts of it. This is due to the periodicity in the signal generated by

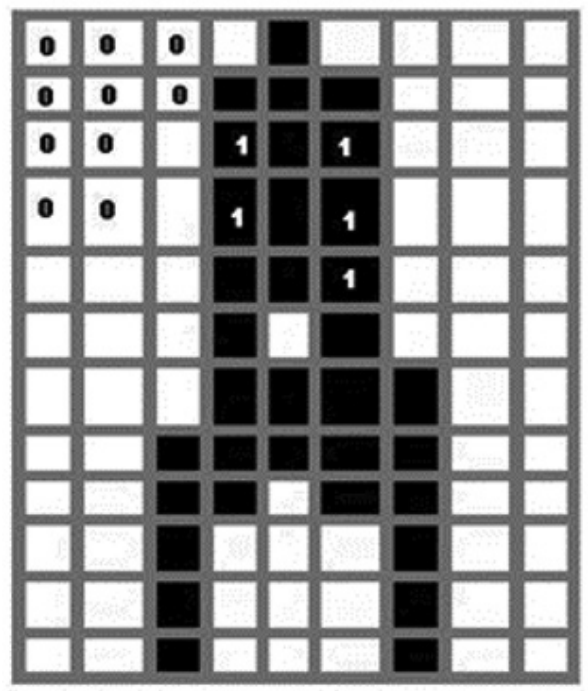

Figure 1: Binary image with foreground pixel $=1$ and background pixel $=0$ 
the characters of the license plate. A set of 5000 images was used as historical data. The images were taken at a parking lot entrance. Each image was visually inspected for the existence of a car in it. For the image signal, the five statistics were considered and used in a statistical model. A Bayesian analysis delivered high probability candidate regions in the image. To locate the license plate in one of the candidate regions, another probabilistic method was used that yielded the achieved accuracy.

\subsection{Probabilistic Optical Character Recognition}

The LPR optical character recognition is the problem where uncertainty prevails as to what class an input image belongs to. Given that it is a stochastic problem, one expects probability answers, if one adheres to the principle that probability is the only coherent way to address uncertainty (Lindley, 1987). Despite some attempts, such as the probabilistic transition trees (Eichelberger and Najarian, 2006), the only noticeable probability based research direction is that of the probabilistic neural networks, for example (Anagnostopoulos, Anagnostopoulos, Loumos and Kayafas, 2006). The probabilistic neural network (PNN) was developed by Donald Specht (1988) and provides a solution to classification problems using Bayesian classifiers and the Parzen Estimators. It is a class of neural networks which combine statistical pattern recognition and feed-forward neural networks technology. It is characterized as having very fast training times and it produces outputs with Bayes posterior probabilities. PNNs are very effective for pattern recognition. However, the LPR character recognition problem is a simple OCR problem and can be addressed by a full probabilistic approach such as the one we present. To solve the problem probabilistically is to treat features or statistics from the input image, using a probability model. The input character image to the OCR module is usually a binary image such as the one in Figure 1 . The historical data of extracted characters is made of two sets; training data and validation data. These are images of license plate characters that have been extracted from images of vehicles using the first two steps LPR. They are binary images that have been cleaned, cropped and normalized (Figure 2). The characters are visually inspected one by one and classified in the 36 possible classes $\{\mathrm{A}, \mathrm{B}, \mathrm{C}, \ldots, \mathrm{X}, \mathrm{Y}, \mathrm{Z}, 0,1, \ldots, 8,9\}$. Each set of characters is then split into a training set and a validation set.

Examples of statistics of an extracted character are the fill percentage and the projected foreground. The fill percentage is the proportion of foreground pixels in the binary

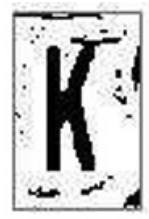

(a)

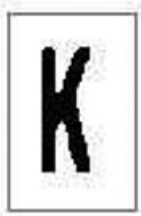

(b)

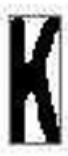

(c)

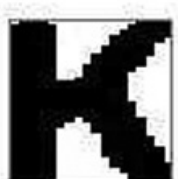

(d)

Figure 2: Extracted character (a), cleaned (b), cropped (c) and normalized (d)
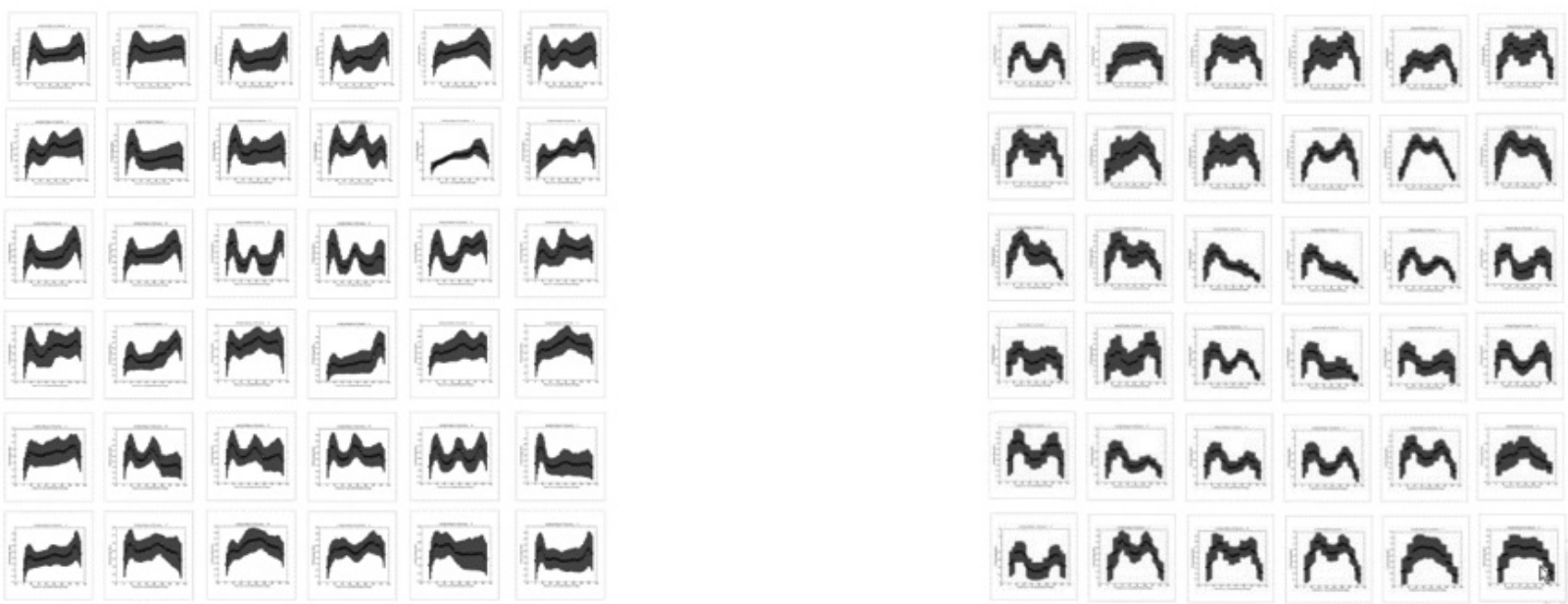

Figure 3: Historical means of projected foreground for the 36 characters 
image. If $F$ is the matrix of the digitized binary image, then the fill percentage is $\sum_{x} \sum_{y} F(x, y) /\left(N_{x} N_{y}\right)$, where $N_{x}$ and $N_{v}$ are the height and width of the image in pixels. The projected foreground is the normalized projection of the foreground on the $x$ and $y$ axes of the image $\sum_{y} F(x, y) / N_{y}$, $x=1, \ldots, N_{x}$ and $\sum_{x} F(x, y) / N_{x}, y=1, \ldots, N_{y}$ respectively. These statistics seem to provide distinguishing information, as seen in Figure 3 where the mean of these statistics is plotted within one standard deviation. We used the minimization of squared errors to attempt to recognize the characters using the normalized projection foreground:

$$
\begin{aligned}
& \text { Minimize }_{(C=A, B, \ldots, X, Y, Z, 0,1, \ldots, 8,9)} \\
& \sum_{x}\left(\sum_{y} F(x, y) / N_{y}-\mu_{x}^{C}\right)^{2}+\sum_{y}\left(\sum_{x} F(x, y) / N_{x}-\mu_{y}^{C}\right)^{2}
\end{aligned}
$$

where $\mu_{x}{ }^{C}$ and $\mu_{y}{ }^{C}, C=A, B, \ldots, X, Y, Z, 0,1, \ldots, 8,9$, are the historical means from the training set. The approach failed to return a good recognition. In an attempt to remedy, the projected foreground was augmented with the distance of the pixels to the side of the image, thus incorporating the information about the locations of the foreground pixels. The minimum squared errors method failed again, an indication that these features of the image do not offer enough information to fully distinguish among the characters.

In general, let $Z$ be the random variable that represents the statistical feature of a character image. For example, $Z$ can be the fill percentage seen above. Note that $Z$ need not, and often isn't univariate. The probabilistic approach starts by building a probability model for that feature in the form of $\operatorname{Prob}(Z \mid C), C=A, B, \ldots, 9$. For each image in the training set, the value of $Z$ is computed. Data analysis tools are used, along with any engineering/prior knowledge to arrive at the probability model $\operatorname{Prob}(Z \mid C)$. Seen as a function of the event $C$, that is the character is $C$, the probability model $\operatorname{Prob}(Z \mid C)$ is known as the likelihood function $\mathrm{L}(\mathrm{C})=\operatorname{Prob}(Z \mid C)$. This likelihood function is at the heart of the probabilistic approach. If this model is built properly and the statistical feature $Z$ offers enough information about the character's class, the probabilistic approach will be effective. Given a likelihood model, the probabilistic approach proceeds as follows. Let $z$ be the value of $Z$ for an image being analyzed. Then the probability that the character is $C$, given the data $z$ is

$$
\operatorname{Prob}(C \mid Z=z)=\frac{\operatorname{Prob}(Z=z \mid C) \operatorname{Prob}(C)}{\sum_{S=A}^{S=9} \operatorname{Prob}(Z=z \mid S) \operatorname{Prob}(S)}
$$

for $C=A, B, \ldots, 9$. $\operatorname{Prob}(C)$ is the model built with any prior knowledge about what $C$ might be. It is called the prior distribution.

$$
\delta=\sum_{S=A}^{S=9} \operatorname{Prob}(Z=z \mid S) \operatorname{Prob}(S)
$$

is a normalizing constant. $\operatorname{Prob}(C \mid Z=z)$ is the posterior distribution of $C$. The solution is given in the selected charac- ter $\hat{C}$, mode of the posterior distribution,

$$
\operatorname{Prob}(\hat{C} \mid Z=z)=\operatorname{Max}_{C=A, B, \ldots, 9} \operatorname{Prob}(C \mid Z=z) \text {. }
$$

If there is no prior knowledge as to what $C$ might be, then the discrete uniform distribution is used where $\operatorname{Prob}(C)=1 / 36$ for $C=A, B, \ldots, 9$. This probabilistic approach is often referred to as a Bayesian approach. A non-Bayesian approach would simply maximize the likelihood function, ignoring the prior component, and select $\hat{C}$ such that

$$
\operatorname{Prob}(\hat{C} \mid Z=z)=\operatorname{Max}_{C=A, B, \ldots, 9} \operatorname{Prob}(Z=z \mid C)
$$

As one can see, both these statistical approaches rely on the likelihood function. These simple operations are at the heart of many probabilistic predictions, classifications and inferences. While seemingly simple in principle, their success depends on the proper selection of the random variable $Z$ and the probability model $\operatorname{Prob}(Z \mid C)$.

In our search for a statistic $Z$, we arrived at the conclusion that the values of the pixels in the binary image hold all the information needed to recognize the character in an image that has been cleaned, cropped and normalized. We defined $Z$ as the multidimensional vector of the values of all the pixels in the image. Each of these values is either 0 or 1 , the input image being binary. For each pixel, we applied the Bernoulli probability model $\theta_{i}^{(Z i}\left(1-\theta_{i}\right)^{I-Z i}$, $Z_{i}$ being the value of $Z$ at pixel $i$. Making the assumption of conditional independence of the pixel values given an image, we construct the likelihood function

$$
L(C)=\operatorname{Prob}(Z \mid C)=\prod_{i=1}^{|Z|} \theta_{i}^{Z_{i}}\left(1-\theta_{i}\right)^{1-Z_{i}}
$$

where $|Z|$ is the cardinal, or vector size, of $Z$. To estimate the proportion $\theta_{i}$ for pixel $i$, a number of approaches are available. But given that the sizes of the historical sets are relatively large, in the order of 200 images per character $C=A, B, \ldots, X, Y, Z, 0,1, \ldots, 8,9$, the estimates converge to the average

$$
\hat{\theta}_{i}=\sum_{j=1}^{N_{C}} x_{i, j} / N_{C}
$$

where $N_{c}$ is the size of the training set for character $C$, and $x_{i j}=\{0$ or 1$\}$ is the value of pixel $i$ for image $j$ of the training set. This is done for each character $C$. For simplicity of exposition, we used $\theta_{i}$ when in fact it is a $\theta_{i}$ (C) that differs for each $C$. From a computational point of view, the assessment of the likelihood parameters is very simple. For each character $C$, all the image matrices of the training set are added, then divided by the size of the set $N_{c}$, automatically providing a matrix of estimates

$$
\left[\hat{\theta}_{i}\right]_{i=1}^{|Z|}
$$




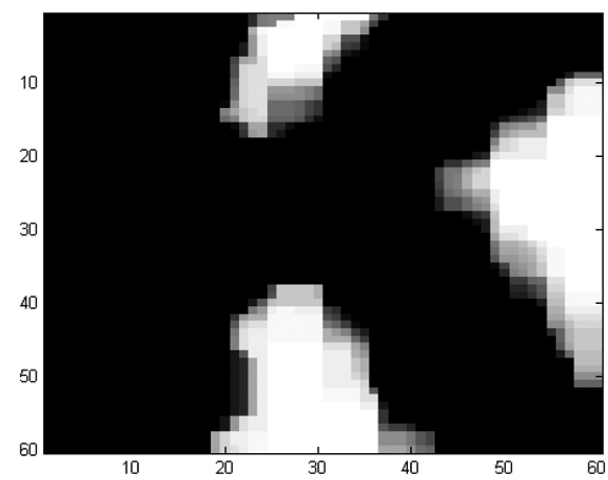

Figure: 4 Likelihood image of character $K$

This is a simple operation, inexpensive computationally, that replaces the training of a neural network. It needs to be done only once, and the estimates matrices are used subsequently to recognize the characters.

Figure 4 shows the matrix

$$
\left[\hat{\theta}_{i}\right]_{i=1}^{|Z|}
$$

for character $\mathrm{K}$. Each value of the pixel $i$ of the image in Figure 4 is the estimate $0 \leq \theta_{i} \leq 1$ for character $K$. It is the estimate of the parameter of the Bernoulli model for pixel $i$, for character $\mathrm{K}$. In essence, this method builds a likelihood function value for each character $C$, that results from a matrix, where the values of 1 signify that the corresponding pixels are always present in the foreground of $C$ (in black in Figure 4), the values 0 mean that the corresponding pixels are always background in $C$ (in white in Figure 4), and the values in between correspond to pixels $i$ that are present in $C$ with a positive probability $\theta$. One observes that the $\mathrm{K}$ in the image is not a perfect one, due precisely to statistical variation. In addition, the colors of the image do not show all the nuances in the values. But the matrix

$$
\left[\widehat{\theta}_{i}\right]_{i=1}^{|Z|}
$$

is computed with double precision accuracy and provides accurate estimates of the probabilities of the foreground existence.

Once the likelihood function is constructed, it is used to recognize characters in a simple operation. Let $z$ be the realization of the statistic $Z$ for a binary image that has received similar cleaning, cropping and resizing as have the images of the training set. Then

$$
\operatorname{Prob}(C \mid z)=\frac{\operatorname{Prob}(z \mid C) \operatorname{Prob}(C)}{\sum_{S=A}^{S=9} \operatorname{Prob}(z \mid S) \operatorname{Prob}(S)}
$$

where

$$
\operatorname{Prob}(z \mid C)=\prod_{i=1}^{|Z|} \hat{\theta}_{i}^{z_{i}}\left(1-\hat{\theta}_{i}\right)^{1-z_{i}}
$$

noting that the appropriate $\theta_{i}^{r}$ correspond to a given $C$. This posterior probability distribution ranks the characters $A, B, \ldots, X, Y, Z, 0,1, \ldots, 8,9$ for their likelihood of being the character in the image being treated. This method provides a full probabilistic approach. One introduces prior knowledge in the Bayesian analysis above in the form of $\operatorname{Prob}(C)$ for each character $C$. Such knowledge can vary from country to country for example, where the position of a character in the license plate may indicate whether it is a letter or a number, for example. If such knowledge does not exist, or is hard to embody in a formal model, then the characters are declared to be equally probable a priori, letting $\operatorname{Prob}(C)=1 / 36$ in this case. To further improve the speed and increase the accuracy, we note that maximizing the product of two bounded positive values is equivalent to maximizing their sum. Therefore we implemented the following

$\operatorname{Max}_{C=A, B, \ldots, 9} f(C)=\sum_{i=1}^{|Z|}\left\{\hat{\theta}_{i}^{Z_{i}}+\left(1-\hat{\theta}_{i}\right)^{1-z_{i}}\right\}$

where $f(C)$ is the score function defined by the sum. This method yielded excellent results.

\subsection{Global Thresholding}

Al-Hmouz and Aboura (2014) used a global thresholding method to improve significantly the performance of a signal processing localization algorithm. This is in contrast to most methods that use a local thresholding approach in view of the fact that global thresholding is hard to perform adequately. The global thresholding method of Aboura (2008) was effective in highlighting the signal of the license plate (Figure 5). A more effective global thresholding method experimented with consisted of the colorbased method of Aboura (2008) coupled with a range of techniques including clustering. In a striking result, all but the characters of the license plate remained as foreground 
in the thresholded image. Such an approach would eliminate the need for a localization algorithm and facilitate greatly the recognition of the characters. In most cases, only some of the characters or part of remained as foreground. This still reduces considerably the localization of the license plate. This research was not completed and is a future direction of work.

\section{Research Results}

\subsection{Object Localization in Image}

In Al-Hmouz and Aboura (2014), a statistical localization approach of Discrete Fourier Transform and five statistics of the plate signal is used to extract the license plate in LPR system. The approach yielded $99 \%$ of license plates were among few candidate regions and the overall performance was $97.27 \%$ for local data set and $88.1 \%$ for public data set. The method is considered computationally complex compared to other methods in the literature. However, the processing time can be reduced to competitive values (150 ms) considering less number of sliding window locations by increasing the step size of the sliding window and ignoring places where license plates do not exist such as image sides. Methods such as CCA and edge detection could be used in improving robustness after the plate candidate regions have been determined. In the future, information about license plate images which are not localized will be studied through suitable models.

\subsection{Probabilistic Optical Character Recognition}

The LPR optical character recognition falls within the realm of pattern recognition. While such problems often require sophisticated approaches due to the large number of patterns, known and unforeseen ones, making it hard to use parsimonious probability models, the recognition problem in LPR is much simpler. We introduced a simple, and very inexpensive method to solve a relatively important problem, using a full probabilistic approach. As most methods in this category, the approach failed to distinguish as such fully between some characters like 2 and Z, 5 and S, 1 and I, B and 8, and $\mathrm{O}, 0, \mathrm{D}$ and Q. However, using the same logic and applying it exclusively to parts of the image, we reach a $97 \%$ reliability. In Figure 6, we show how 2 and $\mathrm{Z}$ are distinguished by focusing the probabilistic OCR algorithm in the areas of the boxes. The likelihood of the pixels in those areas has already been computed, and it is applied to the image that was initially recognized either as a 2 or a Z. Similarly, Figure 7 shows how the remaining confusions are dealt with. This added step is a refinement equivalent to moving a magnifier glass, in the form of the probabilistic OCR algorithm, to parts of the image. Although the likelihood function is the same, the scoring changes and the choice is between two, or four, rather than 36 candidates.

The algorithm was tested on a large validation set, of the order of a couple of hundreds of images per character. Table I shows the reliability per character, summing up to an overall 0.9705 reliability. One could possibly conduct comparison tests with other methods. But as it is often the case in LPR problems, the scenarios and setups that provide sources of data vary dramatically, making it hard to compare universally. We used two sources of data; images

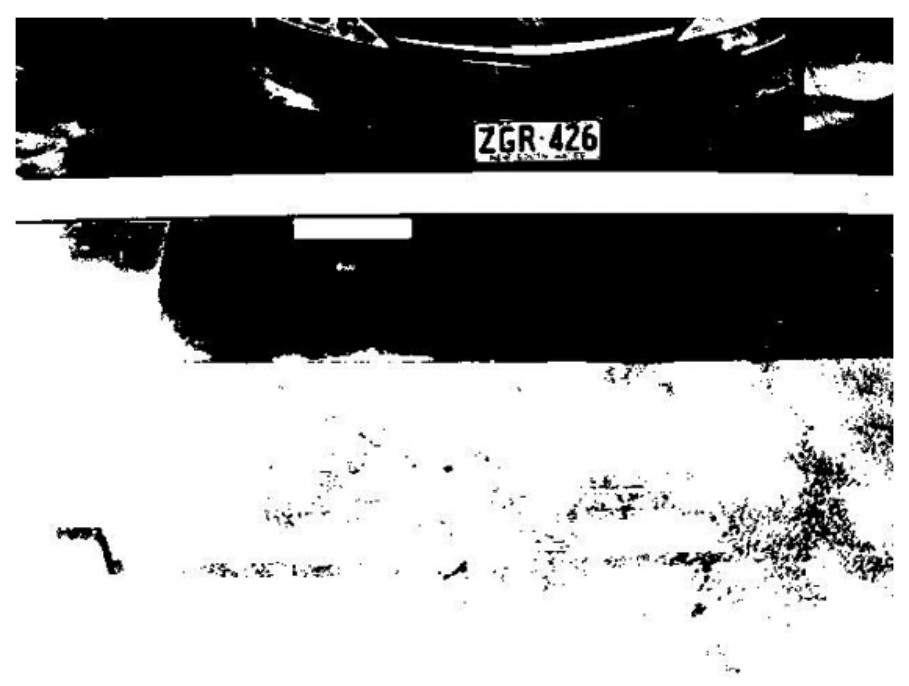

Figure 5: Image thresholded using global thresholding method 

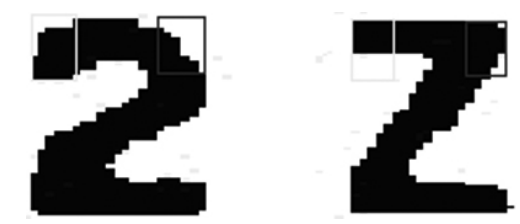

Figure 6: Probabilistic OCR applied to eliminate Confusions

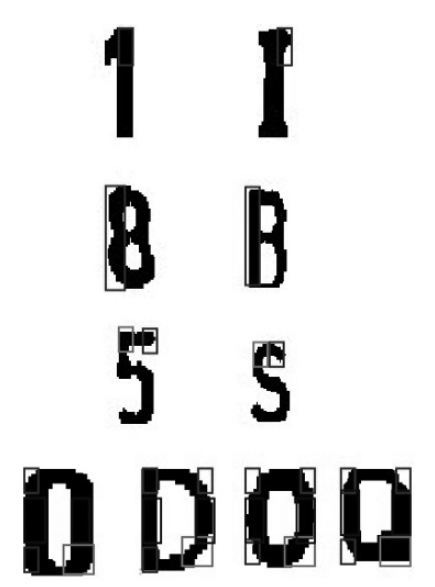

Figure 7: Refinement of the Probabilistic OCR

taken live from a LPR system setup in a parking lot entrance, and images taken from the entrance of the parking lot of the university. The lighting conditions and the positioning of the cameras differed between the two locations. In addition, two slightly different character segmentation methods were used in each case. The tests conducted performed well on both types of data mixed together, as indicated in table I.

\subsection{Global Thresholding}

A global thresholding method for the automatic license plate recognition is introduced that makes use of the colour information in the image through a probabilistic model. LPR still finds it difficult to apply global binarisation whereby a single threshold value for the entire image is calculated. Local thresholding methods are preferred, but their efficiency comes at a cost. Reported work is often focused on one of the three LPR steps, and thresholding is assumed effective. Thresholding a range of images in an automatic fashion is not a trivial task. Aboura (2008) introduces the concept of a statistically determined global threshold for LPR images that is shown to be effective on a large data set. Although the approach is enveloped in a linear regression model, it is the way information from the image is selected and introduced in the explanatory variables that leads to the reliable results. The reported methodology proposes a model that can be used in conjunction with other methods to result in automated systems.

\section{Discussion}

All the previous approaches adopted probabilistic models in LPR steps. Each approach improved the recognition rate for the plate with regard to localization recognition rate and character recognition rate. Using both approaches in LPR system yielded an average recognition rate that reaches up to $97.13 \%$. As mentioned earlier, the confusion between some characters (refer to figure 6 and 7) might have the major drawback of this algorithms. However, this problem will not have an effect on bilingual plates in which two independent texts are appeared in the plates.

In some non-English speaking countries like Saudi Arabia, license plate appears in English and Arabic text (Figure 8). Arabic letters are associated with their corresponding English letters. This can be considered as two source of information available for character recognition. In this case, the probabilities of Arabic and English characters can be combined in character recognition phase. The posterior of each Arabic characters is fused with its associated English characters. The prevailing character probabilities after fusion will be considered as the final recognition. The flow chart of license plate recognition for bilingual plates is show in Figure 9. 
Table 1: OCR Reliability Results

\begin{tabular}{|c|c|c|c|}
\hline Character & Reliability & Character & Reliability \\
\hline A & 0.9888 & S & 0.9385 \\
\hline B & 0.9259 & T & 0.9828 \\
\hline C & 0.9841 & U & 1.0000 \\
\hline D & 0.9552 & V & 0.9306 \\
\hline E & 0.9359 & W & 0.9898 \\
\hline F & 1.0000 & X & 1.0000 \\
\hline G & 0.9608 & Y & 1.0000 \\
\hline H & 1.0000 & Z & 0.9722 \\
\hline I & 0.9500 & 0 & 0.8636 \\
\hline J & 1.0000 & 1 & 0.8864 \\
\hline K & 1.0000 & 2 & 1.0000 \\
\hline L & 1.0000 & 3 & 0.9649 \\
\hline M & 1.0000 & 4 & 0.9899 \\
\hline N & 0.9529 & 5 & 0.9623 \\
\hline O & 0.9630 & 6 & 0.9756 \\
\hline
\end{tabular}
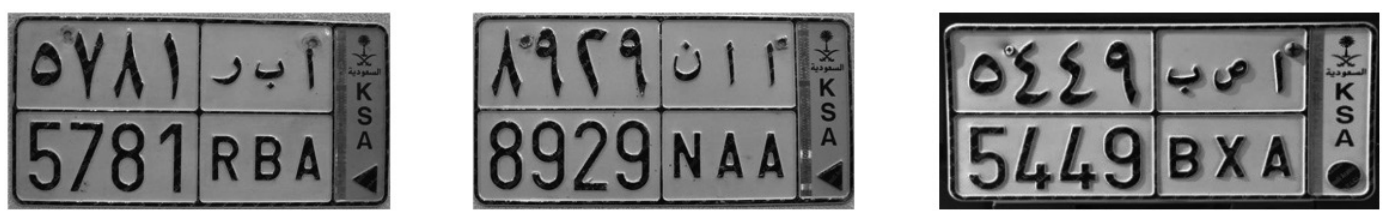

Figure 8: Saudi Arabia license plate examples

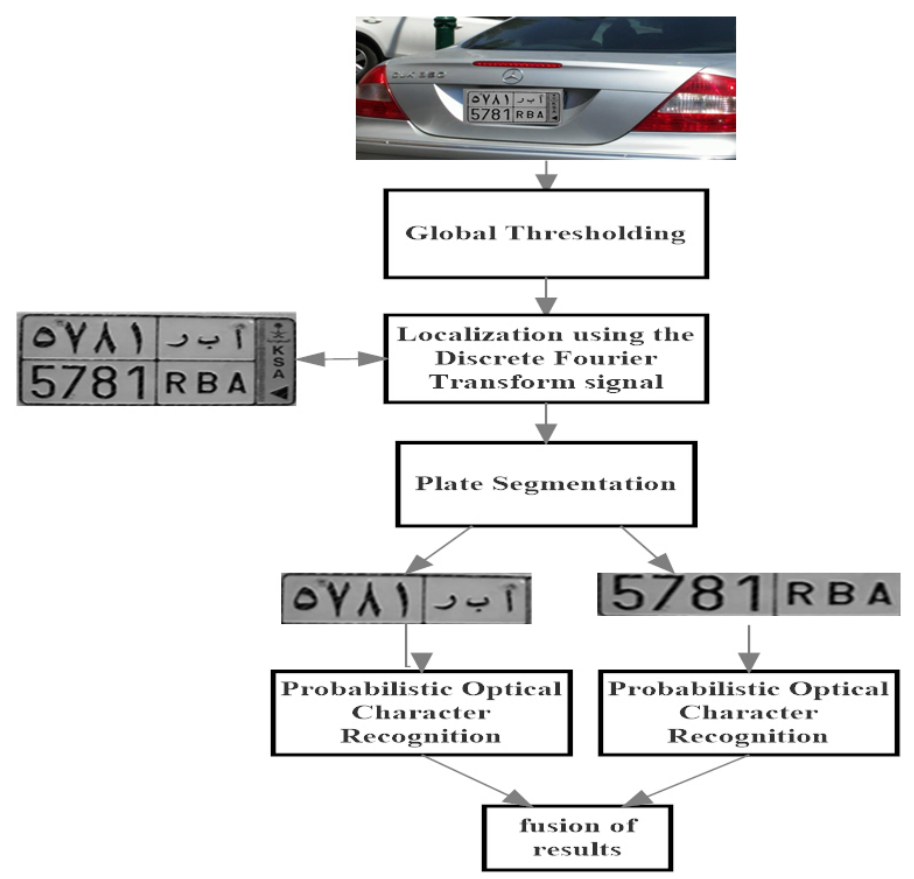

Figure 9: Bilingual plate (Arabic English) processing steps 


\section{Conclusions}

In this paper, we described a number of solutions that can be used in recognition systems for decision support. These algorithms are effective alongside the application of global thresholding and other techniques that uses frequency of signals from the images by means of the Discrete Fourier Transform. This paper presented a probabilistic character recognition approach. The likelihood functions were formed using the Bernoulli model for each pixel value and the posterior probabilities of characters were obtained by fusing the probabilities of pixel value in each location. In making the assumption of independence between pixels of the image in the construction of the likelihood function, we in fact constructed a Naïve Bayes solution. The results showed an impressive recognition rate of average $(97.13 \%)$ when using the previous approaches. Also an improvement of recognition rate can be achieved when there two source of information especially of license plates that have two independent texts. For further study, fusion process at pixel level for bilingual plates can investigated.

\section{References}

Aboura, K. (2008). Automatic Thresholding of license plate. International Journal of Automation and Control, 2(2-3), pp. 213-231. DOI: 10.1504/ IJAAC.2008.022178.

Aboura, K. \& R. Al-Hmouz, R. (2007). Probabilistic license plate optical character recognition. School of Computing and Communication, University of Technology Sydney. (Technical Report K-OCR-2-07)

Acosta B.D. (2004) Experiments in image segmentation for automatic US license plate recognition. Master's thesis, Virginia Polytechnic Institute and State University.

Al-Hmouz, R., \& Aboura, K. (2014). License plate localization using a statistical analysis of discrete fourier transform signal. Computers and Electrical Engineering, 40(3), pp. 982-992, http://dx.doi.org/10.1016/j. compeleceng.2014.01.001

Anagnostopoulos, C., Kayafas, E., \& Loumos, V. (2000). Digital image processing and neural networks for vehicle license plate identification. Journal of Electrical Engineering, 1(2), pp. 2-7.

Anagnostopoulos, C., Anagnostopoulos, I., Loumos, V., $\&$ and Kayafas, E. (2006). A license plate recognition algorithm for intelligent transportation system applications. IEEE Transactions on Intelligent Transportation Systems, 7(3), pp. 377-392, http://dx.doi.org/10.1109/ TITS.2006.880641

Canny, J. (1986). A computational approach to edge detection. IEEE Transactions on Pattern Analysis and Machine Intelligence, 8 (6), pp. 679-698, http://dx.doi.
org/10.1109/TPAMI.1986.4767851

Chang S.L, Chen. LS, Chung Y.C, Chen S.W. (2004). Automatic license plate recognition. IEEE Trans Intell Transport Syst, (1), pp. 42-53, http://dx.doi. org/10.1109/TITS.2004.825086

Dlagnekov L. (2004). License plate detection using AdaBoost. Comput. Sci. Eng. Dept., Univ. California, San Diego, CA; March 2004. Retrieved from http://www. cse.ucsd.edu/classes/fa04/cse252c/projects/louka.pdf

Duc DT, Hong T.L, Vinh P.T, Viet H.N. (2005). Building an automatic vehicle license plate recognition system. In Proc. Intl. Conf. in Computer Science - RIVF'05 February 21-24, 2005, Can Tho, Vietnam, pp. 59-63.

Eichelberger, C.N., \& Najarian, K. (2006). Simulating protein computing: character recognition via probabilistic transition trees. IEEE International Conference on Granular Computing, pp. 101-105, http://dx.doi. org/10.1109/GRC.2006.1635766

Horowitz, M. (1957). Efficient use of a picture correlator. Journal of the Optical Society of America, 47(4), pp. 327-327, http://dx.doi.org/10.1364/JOSA.47.000327

Lindley, D.V. (1987). The probability approach to the treatment of uncertainty in artificial intelligence and expert systems. Statistical Science, 2(1), pp. 17-24, http://dx.doi.org/10.1214/ss/1177013427

Kim KK, Kim KI, Kim JB, Kim HJ. (2000). Learning-based approach for license plate recognition. In: Proc. 2000 IEEE signal processing society workshop, Sydney, Australia, pp. 614-23.

Otsu, N. (1979). A threshold selection method from gray-level histogram. IEEE Transactions on System, Man and Cybernetics, 9(1), pp. 62-66, http://dx.doi. org/10.1109/TSMC.1979.4310076

Pratt, W.K. (1974). Correlation techniques of image registration. IEEE Transactions on Aerospace and Electronic Systems, 10, pp. 353-358, http://dx.doi.org/10.1109/ TAES.1974.307828

Specht, D.F. (1988). Probabilistic neural networks for classification, mapping or associative memory. IEEE International Conference on Neural Networks, 1, pp. 525-532, http://dx.doi.org/10.1109/ICNN.1988.23887 
Khalid Aboura, PhD, teaches quantitative methods at the College of Business Administration, University of Dammam, Saudi Arabia. Khalid Aboura spent several years involved in academic research at the George Washington University, Washington D.C, U.S.A. He worked as a Research Scientist at the Commonwealth Scientific and Industrial Research Organization of Australia and conducted research at the University of Technology Sydney. Khalid Aboura was a Scientist at the Kuang-Chi Institute of Advanced Technology of Shenzhen, China.
Rami Al-Hmouz received his $\mathrm{PhD}$ degree in computer engineering from the University of Technology, Sydney, Australia, in 2008. Currently, he is an Assistant Professor in Department of Electrical and Computer Engineering at King Abdulaziz University in Saudi Arabia. His research interests are in machine learning, fuzzy logic, granular computing, and computer vision. 